\title{
Simplified procedure for the routine isolation of Clostridium difficile from faeces
}

\author{
SAVERIO P BORRIELLO, PAULINE HONOUR \\ From the Division of Communicable Diseases, Clinical Research Centre, Watford Road, Harrow, \\ Middlesex, HAl $3 U \mathrm{UJ}$
}

SUMmARY The use of alcohol, at a final concentration of $50 \%$, as a selective procedure for the isolation of Clostridium difficile was compared to a selective medium containing $250 \mu \mathrm{g} / \mathrm{ml} \mathrm{of}$ cycloserine and $10 \mu \mathrm{g} / \mathrm{ml}$ of cefoxitin. Of 266 faecal samples 82 were shown to be positive by one or other method. Seventy-seven $(94 \%)$ of these were detected by the selective agar (SA) and $72(88 \%)$ by the alcohol procedure (AP). Ten samples $(12 \%)$ were positive only by SA and five samples $(6 \%)$ by AP only. The AP was further modified so that all manipulations prior to incubation were performed on the open bench. Of 18 positive samples, $18(100 \%)$ were detected by SA and $16(89 \%)$ by AP.

There has been an increasing body of evidence generated during the last few years which firmly implicates Clostridium difficile as the aetiological agent of both antibiotic and non-antibiotic associated pseudomembranous colitis (PMC). ${ }^{1-4}$ There is also recent evidence to implicate this microorganism in diarrhoea unrelated to $\mathrm{PMC}^{4}$ and as a possible factor in some exacerbations of inflammatory bowel disease.$^{56}$ As a result of these findings, an increasing number of laboratories are receiving requests to analyse stools for the presence of $C$ difficile. These investigative procedures were greatly facilitated by the development of a sensitive selective agar incorporating cycloserine and cefoxitin as selective agents. ${ }^{78}$ The purpose of this study was to evaluate a simplified procedure for the isolation of $C$ difficile from stools that was independent of a selective agar, and to further modify this procedure so that it was also independent of sophisticated anaerobic facilities.

This study was divided into two parts. The first part was concerned with the evaluation of the use of alcohol to select for clostridial spores as a selective procedure (alcohol shock), compared to a selective antibiotic-containing medium, for the recovery of $C$ difficile from faeces. The second part of the study evaluated a modification of this procedure that employed the minimum of anaerobe culture facilities (bench procedure).

Accepted for publication 6 April 1981

\section{Material and methods}

MATER I A L

\section{Source of specimens}

The 266 specimens used in the study were forwarded to this laboratory from a number of hospitals throughout the United Kingdom to be investigated for the presence of $C$ difficile or its associated cytotoxin, or both. All samples were processed immediately on receipt.

\section{Brain-heart infusion agar $(B H I A)$}

All consitutuents are expressed as grams or millilitres per litre of distilled water. Difco brain heart infusion $37 \mathrm{~g}$; Difco yeast extract $5 \mathrm{~g}$; haemin $ᄋ$ solution $1 \mathrm{ml}$; vitamin $\mathrm{K}_{1}$ solution $1 \mathrm{ml}$; L-cysteine HCL $0.5 \mathrm{~g}$; sodium formaldehyde sulphoxylate $ᄋ$ $0.3 \mathrm{~g}$; New Zealand agar $20 \mathrm{~g}$; defribrinated horse blood $100 \mathrm{ml}$; and Tween $801 \mathrm{ml}$.

Cycloserine-cefoxitin agar (CCA)

Cycloserine (Sigma) and cefoxitin (Merck Sharp and $N$ Dohme) at concentrations of $250 \mu \mathrm{g}$ and $10 \mu \mathrm{g}$ per $\mathrm{ml} \underset{\mathrm{C}}{\mathrm{N}}$ respectively were added as selective agents ${ }^{8}$ to the BHIA.

\section{Haemin solution}

One gram of haemin was dissolved in $5 \mathrm{ml}$ of $1 \mathrm{~N}$ $\mathrm{NaOH}$ and then made up to $100 \mathrm{ml}$ with distilled water. 
Vitamin $K_{1}$ solution

One gram of Vitamin $K_{1}$ was dissolved in $99 \mathrm{ml}$ of absolute ethanol.

\section{ISOLATION PROCEDURES}

Three different methods were employed to isolate $C$ difficile. Samples of stool were diluted in glycerol transport broth ${ }^{9}$ to yield a $1 / 10$ dilution. This dilution was passed into an anaerobic chamber. Serial tenfold dilutions over four steps were prepared in the chamber with prereduced brain-heart infusion broth (BHIB). ${ }^{10}$

(i) Selective agar A sample of $0.1 \mathrm{ml}$ of the serial dilutions was plated on to CCA medium which had been stored at room temperature under an anaerobic environment for at least two days before use.

(ii) Alcohol shock Of the first stool dilution, 0.5 $\mathrm{ml}$ was added to an equal volume of absolute ethanol which had been sterilised by tyndallisation ${ }^{11}$ and mixed on a vortex mixer. After being allowed to stand at room temperature for $1 \mathrm{~h}$ serial tenfold dilutions over four steps were prepared in prereduced $\mathrm{BHIB}$, and samples of $0.1 \mathrm{ml}$ of the serial dilutions were plated on to BHIA medium which had been stored at room temperature under an anaerobic environment for at least two days before use. All procedures were performed in the anaerobic cabinet.

(iii) Bench alcohol shock procedure This procedure was as described for the alcohol shock procedure described above with the exceptions that all procedures were performed on the open bench and none of the reagents used were prereduced. Samples of $0.1 \mathrm{ml}$ of the serial dilutions were plated on to routine aerobic $5 \%$ blood Columbia agar plates, and no attempt was made to prereduce the medium in any way.

\section{INCUBATION PROCEDURES}

Both CCA and BHIA plates were incubated at $37^{\circ} \mathrm{C}$ under an atmosphere of $10 \% \mathrm{CO}_{2}, 10 \% \mathrm{H}_{2}$ and $80 \%$ $\mathrm{N}_{2}$ for at least $48 \mathrm{~h}$, in an anaerobic incubator housed in the anaerobic cabinet. Inoculated blood plates from the bench alcohol shock procedure were placed in a conventional anaerobe jar. Anaerobiosis was achieved by the use of a "gas generating kit" system (Oxoid). The anaerobe jar was placed in an incubator at $37^{\circ} \mathrm{C}$, and the plates incubated for at least $48 \mathrm{~h}$.

\section{IDENTIFICATION CRITERIA}

After incubation all plates were examined under long wave $(360 \mathrm{~nm}$ ) ultraviolet light (UV L-25 Mineralight Lamp. Ultra-violet products Ltd, Cambridge), for the low green/chartreuse fluorescence characteristic of $C$ difficile colonies. ${ }^{7}$ A count of all presumptive $C$ difficile colonies was made and at least three of these colonies when present from each of the plates were subcultured into Robertsons Cooked Meat (Southern Group Laboratories). After three days incubation the cultures were checked for purity and identified by use of the volatile fatty acid profile of carbohydrate metabolism generated by gas-liquid chromatography and biochemical reaction patterns. ${ }^{12}$ All isolates were screened for their ability to produce cytotoxin.

\section{CYTOTOXIN ASSAY}

Isolates were analysed for the ability to produce a cytotoxin that was specifically neutralised by $C$ sordellii antitoxin (Wellcome Research Laboratories) by the "conventional tube method" described in a previous publication. ${ }^{13}$ All stool samples were also analysed for the presence of this toxin.

\section{Results}

Comparison of CCA selective agar and alcohol shock procedure

The results are presented in the Table. Of 266 faecal samples analysed, 82 were shown to have $C$ difficile present by a combination of the two methods described. Of these 82 positive specimens, $77(94 \%)$ were detected by CCA and $72(88 \%)$ by the alcohol shock procedure. Ten samples $(12 \%)$ yielded $C$ difficile on CCA only, and five specimens $(6 \%)$ only by the alcohol shock procedure.

Comparison of methods for recovery of C Difficile from stools

\begin{tabular}{lclll}
\hline Method & No tested & $\begin{array}{l}\text { Total no of } \\
\text { CDifficile } \\
\text { positive stools* }\end{array}$ & $\begin{array}{l}\text { No of stools } \\
\text { yielding } \\
\text { C Difficile (\%) }\end{array}$ \\
\hline $\begin{array}{l}\text { CCA selective } \\
\text { agar }\end{array}$ & 266 & 82 & 77 & $94 \dagger$ \\
$\begin{array}{c}\text { Alcohol shock } \\
\text { Bench alcohol } \\
\text { shock }\end{array}$ & 266 & 82 & 72 & 88 \\
\hline
\end{tabular}

*No of stools shown to harbour $C$ difficile by a combination of all the methods described.

†Expressed as a percentage of total $C$ difficile positive stools.

\section{CCA positive alcohol shock negative}

Seven of the 10 faecal samples that yielded $C$ difficile only on CCA had no demonstrable cytotoxin present, and one of these yielded only non cytotoxigenic $C$ difficile strains. In addition, one of these seven samples came from a patient from whom previous samples were positive for stool cytotoxin and for $C$ difficile by both methods employed. Of the remaining three samples from this set two were stool cytotoxin-positive and one had a cytotoxin that was not neutralised by the cross-reacting $C$ sordellii antitoxin. One of these cytotoxin-positive stools 
came from a patient from whom previous samples yielded $C$ difficile by both methods employed.

In two cases the numbers of $C$ difficile present as determined by CCA (100 organisms per gram of stool) were below the limit of detection for the alcohol shock procedure which is 200 organisms per gram due to the additional 1 in 2 dilution that this procedure requires.

Alcohol shock procedure positive, CCA negative Two of the five specimens in this category were negative for stool cytotoxin. However, the other three cases were stool cytotoxin-positive.

\section{QUANTITATIVE RECOVERY}

In all, 44 specimens were analysed for total counts of $C$ difficile. The CCA selective agar procedure yielded higher counts of $C$ difficile than the alcohol shock procedure in 31 cases $(70 \%)$, but lower counts in 11 cases $(25 \%)$. In two instances, these two procedures yielded the same number of organisms $(5 \%)$. The results demonstrate that more organisms can be recovered by the CCA selective agar method than by the alcohol shock procedure. Analysis of the data by a two-tailed $t$ test showed that this difference was highly significant $(\mathrm{p}<0.001)$ with a $t$ value for 43 degrees of freedom of 3.477 .

COMPARISON OF CCA SELECTIVE AGAR AND BENCH ALCOHOL SHOCK PROCEDURE

The results are presented in the Table. Of 55 faecal samples analysed, 18 were shown to have $C$ difficile present by a combination of the two methods described. Of these 18 positive specimens, $18(100 \%)$ were detected by CCA and $16(89 \%)$ by the alcohol shock procedure. Two samples $(11 \%)$ yielded $C$ difficile on CCA only. In both of these cases the numbers of $C$ difficile detected were small with values of $10^{3 \cdot 4}$ and $10^{2 \cdot 0}$.

CONCORDANCE OF STOOL CYTOTOXIN AND

$C$ DIFFICILE ISOLATION

Cytotoxin that was neutralised by $C$ sordellii antitoxin was detected in 61 of the 266 samples analysed. Sixty of these 61 cytotoxin-positive stools (98\%) were shown to have $C$ difficile present by a combination of the methods being evaluated. Of these, two samples yielded $C$ difficile only by the CCA selective agar and three samples only by the alcohol shock procedure. All of the cytotoxin-positive stools of the 55 used to evaluate the bench alcohol shock procedure yielded $C$ difficile by both the alcohol and selective agar methods employed. Overall, the CCA selective agar detected $C$ difficile in 57 of the 61 cytotoxin-positive stools $(93 \%)$ and the alcohol shock procedure in 58 of these stools $(95 \%)$.

\section{Discussion}

It is now widely accepted that $C$ difficile is the aetiological agent of antibiotic-associated pseudomembranous colitis ${ }^{1-4}$ and also of pseudomembranous colitis unrelated to known chemotherapy. ${ }^{414}$ Information is now also available to implicate the organism and its toxin in antibioticassociated diarrhoea. 415 There have also been two recent reports implicating this organism in exacerbation of inflammatory bowel disease; $;^{6}$ however, these latter associations have not been noted in other studies. ${ }^{115}$ This increasing awareness of the potential pathogenicity of $C$ difficile, especially in the compromised host, has led to a rapid increase in the numbers of laboratories receiving requests to screen for this organism. Although there is a good correlation between the presence of $C$ difficile and stool cytotoxin $^{4}$ this assay requires tissue cultures which are not readily available to many clinical laboratories. The development of a sensitive selective agar ${ }^{7}$ has greatly facilitated the isolation of $C$ difficile. However, not all microbiology laboratories have sophisticated anaerobic, or media preparation facilities. The data presented here indicate that the use of alcohol as a selective procedure is an efficient method for the detection and isolation of $C$ difficile from stools and in particular those containing cyto toxin. In addition, the results indicate that this method can be successfully performed on the bench with the minimum of anaerobic microbiology equipment.

The ability of $C$ difficile to fluoresce under long wave UV light noted by George et al. on their cycloserine-cefoxitin fructose egg yolk agar is also apparent on a blood base agar. ${ }^{7}$ This characteristic can therefore be utilised to aid in the detection and presumptive identification of this organism when isolated by the alcohol shock procedures, and is especially useful to those who are unfamiliar with the colonial characteristics of $C$ difficile. We have not yet isolated any other species of clostridium that exhibits this characteristic. An advantage of the alcohol shock procedure is that there is a normal cellular morphology to the organisms present. The cellular morphology of $C$ difficile can be characteristic, with apparent bipolar sporing noted on Gram-stain of 3-day-old cultures, giving a "dumbbell" appearance, an additional feature which can aid in the detection and presumptive identification of this organism. This contrasts to cells present on media containing cycloserine and cefoxitin as selective agents, where spores are not present. An additional advantage of the alcohol shock procedure is that many other clostridial species are concomitantly isolated. This sort of screening procedure 
should therefore increase the likelihood of noting associations between carriage of $C$ difficile and other clostridial species that may enhance its pathogenic effect. This sort of enhancement has been noted for $C$ tertium and $C$ perfringens in $C$ difficile-mediated diarrhoea in axenic neonatal hares. ${ }^{16}$ The recent finding by Schwartz et al. ${ }^{17}$ implicating toxigenic $C$ perfringens type $\mathrm{C}$ in an ampicillin-induced enterocolitis also highlights the value of an isolation procedure that selects for other clostridial species in addition to $C$ difficile.

In conclusion, we believe that the alcohol shock procedures described are a valid method for the isolation and detection of $C$ difficile from stools and if adopted will greatly increase the number of laboratories that will be able to screen for this pathogen.

The authors wish to thank Judith Robinson for performing the toxin assays and SP Borriello wishes to acknowledge the support of Upjohn International.

\section{References}

${ }^{1}$ Larson HE, Price AB, Honour P, Borriello SP. Clostridium difficile and the aetiology of pseudomembranous colitis. Lancet 1978; i:1063-6.

${ }^{2}$ Bartlett JG, Chang TW, Gurwith M, Gorbach SL, Onderdonk AB. Antibiotic-associated pseudomembranous colitis due to toxin producing clostridia. $N$ Engl J Med 1978;298:531-4.

${ }^{3}$ George RH, Symonds JM, Dimock F, et al. Identification of Clostridium difficile as a cause of pseudomembranous colitis. Br Med J 1978; i 695.

${ }^{4}$ Borriello SP, Larson HE. Antibiotic and pseudomembranous colitis. J Antimicrob Chemother 1981;7, Suppl A : 53-62.

${ }^{5}$ La Mont JT, Trnka YM. Therapeutic implications of Clostridium difficile toxin during relapse of chronic inflammatory bowel disease. Lancet 1980;i:381-3.
[ ${ }^{6}$ Bolton RP, Sherriff RJ, Read AE. Clostridium difficile associated diarrhoea: a role in inflammatory bowel disease? Lancet 1980;i:383-4.

7 George WL, Sutter VL, Citron D, Finegold SM. Selective and differential medium for isolation of Clostridium difficile. J Clin Microbiol 1979;9:214-9.

${ }^{8}$ Willey SH, Bartlett JG. Cultures for Clostridium difficile in stools containing a cytotoxin neutralised by Clostridium sordellii antitoxin. J Clin Microbiol 1979;10:880-4.

${ }^{9}$ Crowther JS. Transport and storage of faeces for bacteriological examination. J Appl Bacteriol 1971;34: 477-83.

${ }^{10}$ Borriello SP, Hudson MJ, Hill MJ. Investigation of the gastrointestinal bacterial flora. Clin Gastroenterol 1978; 7, 2:329-49.

${ }^{11}$ Cruickshank R, ed. Medical microbiology: a guide to the laboratory diagnosis and control of infection. 11th ed. Edinburgh and London: E \& S Livingstone Ltd, 1969: 687.

12 Holdeman LV, Cato EP, Moore WEC, eds. Anaerobic laboratory manual 4th ed. Blacksburg, Virginia: Virginia Polytechnic Institute and State University, 1977.

${ }^{13}$ Larson HE, Price AB. Pseudomembranous colitis: Presence of clostridial toxin. Lancet 1977;ii:1312-4.

14 Peikin SR, Galdibini J, Bartlett JG. Role of Clostridium difficile in a case of non antibiotic-associated pseudomembranous colitis. Gastroenterology 1980;79:948-51.

15 Bartlett JG, Moon N, Chang TW, Taylor N, Onderdonk AB. Role of Clostridium difficile in antibiotic-associated pseudomembranous colitis. Gastroenterology 1978;75: 778-82.

16 Dabard J, Dubos F, Martinet L, Ducluzeau R. Experimental reproduction of neonatal diarrhoeae in young gnotobiotic hares simultaneously associated with Clostridium difficile and other clostridium strains. Infect Immun 1979;24:7-11.

17 Schwartz JN, Hamilton JP, Fekety R, et al. Ampicillininduced enterocolitis: Implication of toxigenic Clostridium perfringens type C. J Pediatr 1980;97:661-3.

Requests for reprints to: Dr SP Borriello, Division of Communicable Diseases, Watford Road, Harrow, Middlesex HA1 3UJ, England. 